\title{
Advancements in biomedical applications of infrared imaging
}

\section{Introduction}

Infrared imaging permits to map the surface thermal distribution of the human body. A large number of studies has been so far established to assess the contribution that such information may provide to the clinicians. Cutaneous temperature distribution depends on complex relationships defining the heat exchange processes between cutaneous tissue, inner tissue, local vasculature, and metabolic activity. All of these processes are mediated and regulated by the sympathetic and parasympathetic activity to keep the thermal homeostasis. At a local level, diseases and disorders can affect the heat balance or exchange processes resulting in modification of the cutaneous temperature and of its functional with respect to healthy conditions. Therefore, the characteristic parameters modeling the spontaneous activity of the cutaneous thermoregulatory system can be used as quantitative diagnostic parameters. The functional Infrared Imaging (fIRI)also named Infrared Functional Imaging (IRFI) - is the study for diagnostic purposes, based on the modeling of the bioheat exchange processes, of the functional properties and alterations of the human thermoregulatory system. In this paper, we will review some of the most important recent clinical applications of the functional infrared imaging.

\section{Quantifying the Relevance and Stage of Disease with the $\tau$ Image Technique.}

Infrared imaging can provide diagnostic information according different possible approaches. The approach generally followed consists of the detection of significant differences between the cutaneous thermal distributions of the two hemisoma or in the pattern recognition of specific features with respect to average healthy population [1]. More valuable and quantitative information can be obtained from the study of the cutaneous temperature dynamics in the unsteady state, where the processes involved and controlled by the thermoregulatory system can be modeled and described through their characteristic parameters [2-7]. The presence of diseases interfering with the cutaneous thermoregulatory system can be then inferred by the analysis of its functional alterations [8-18]. Merla et al. [7, 17, 20, and 21] proposed a new imaging technique, based on this approach, for the clinical study of a variety of diseases. Starting from a general energy balance equation, they have demonstrated that the recovery time from any kind of thermal stress for a given region of interest depends from the region thermal parameters.

The proposed model accept an exponential solution and suggests to use the time constant $\tau$ as a characterizing parameter for the description of the recovery process after any kind of controlled thermal stress, with $\tau$ mainly determined by the local blood flow and thermal capacity of the tissue.To pictorially describe the effect of the given disease, an image reporting pixel to pixel the $\tau$ recovery time can be used to characterize that disease [7, 17, 20, and 21].

The $\tau$ image technique has been first proposed as complementary diagnostic tool for the diagnosis of muscular lesions, Raynaud's phenomenon and Deep Vein Thrombosis [7, 17 20, 21].

\section{Raynaud's Phenomenon and Scleroderma}

Raynaud's phenomenon (RP) is defined as a painful vasoconstriction - that may follow cold or emotional stress - of small arteries and arterioles of extremities, like fingers and toes. RP can be primary (PRP) or secondary (SSc) to scleroderma. None of the physiological measurement techniques currently in use, but infrared imaging, are completely satisfactory in focusing primary or secondary RP [3]. Thermography protocols [3-5, 24-28] usually include cold patch testing to evaluate the capability of the patient hands to re-warm. The pattern of the re-warming curves is usually used to depict the underlying structural diseases. Merla et al. $[14,16]$ proposed study the natural response of the fingertips to exposure to a cold environment through a simple bioheat model to compute the amount of heat produced by the local thermoregulatory system and stored in the fingertip as a possible diagnostic parameter. Such a parameter has been used in $[14,16]$ to discriminate and classify PRP, SSc and healthy subjects on a set of 40 (20 PRP, 20 SSc) and 18 healthy volunteers.

The sensitivity of the method in order to distinguish patients from normal is $100 \%$. The specificity in distinguishing SSc from PRP is $95 \%$.

\section{Diagnosis of Varicocele and Follow Up of the Treatment}

Varicocele is a widely spread male disease consisting into a dilatation of the pampiniform venous plexus and of the internal spermatic vein. Consequences of such a dilatation are an increase of the scrotal temperature and a possible impairment of the potential fertility $[36,37]$. fIRI has been used to determine whether altered scrotal thermoregulation is related to subclinical varicocele [15]. In a study conducted in 2001, Merla and Romani enrolled 60 asymptomatic volunteers that underwent to clinical examination, echo color Doppler imaging (the gold standard) and fIRI. The latter technique accurately detected 22 no symptomatic varicocele. The control of the scrotum temperature should improve after varicocelectomy as a complementary effect of the reduction of the blood reflux. Moreover, follow-up of the changes in scrotum thermoregulation after varicocelectomy may provide early indications on possible relapses of the disease. To answer the above questions, Merla et al. [9] used fIRI to study changes in the scrotum thermoregulation of 20 patients that were judged eligible for varicocelectomy on the basis of the combined results of the clinical examination, Echo color Doppler imaging, and spermiogram. fIRI documented the changes in the thermoregulatory control of the scrotum after the treatment and proved that the surgical treatment of the varicocele induces modification in the thermoregulatory properties of the scrotum, reducing the basal temperature of the affected testicle and pampiniform plexus, and slowing down its recovery time after thermal stress. 


\section{Conclusions}

fIRI is a biomedical imaging technique that relies on high resolution infrared imaging and on the modeling of the heat exchange and control processes at the cutaneous layer. fIRI is aimed to provide further information about the studied disease to the physicians, like explanation of the possible physics reasons of some thermal behaviors and their relationships with the physiology of the involved processes. One of the great advantages of fIR imaging is the fact that is not invasive and it is a touchless imaging technique. fIR is not a static imaging investigation technique. Therefore, data for fIR imaging need to be processed adequately for movement. Adequate bio heat modeling is also required. The medical fields for possible applications of fIR imaging are numerous, ranging from those described into this chapter, to psychometrics, cutaneous blood flow modeling, peripheral nervous system activity, and some angiopathies. The applications described in this paper show that fIR imaging provides highly effective diagnostic parameters. The method is highly sensitive, but also highly specific into discriminating different conditions of the same disease.

\section{References}

Aweruch, M.S., Thermography: its current diagnostic status in muscular-skeletal medicine, Med. J. Aust, 154, 441, 1991.

Prescott, et at., Sequential dermal microvascular and perivascualr changes in the development of scleroderma, J. Pathol., 166, 255, 1992.

Herrick, A.L. and Clark, S., Quantifying digital vascular disease in patients with primary Raynaud's phenomenon and systemic sclerosis, Ann. Rheum. Dis., 57,70, 1998.

Darton, K. and Black, C.M., Pyroelectric vidicon thermography and cold challenge quantify the severity of Raynaud's phenomenon, $\mathrm{Br} . \mathrm{J}$. Rheumatol., 30,190,1991.

Javanetti, S., et al., Thermography and nailfold capillaroscopy as noninvasive measures of circulation in children with Raynaud's phenomenon, J. Rheumatol., 25, 997,1998.

Merla, A., et al., Dynamic digital telethermography: A novel approach to the diagnosis of varicocele, Med. Biolog. Eng. Comp., 37,1080, 1999.

Merla, A., et al., Correlation of telethermographic and ultrasonographic reports in the therapeutic monitoring of second-class muscular lesions treated by hyperthermia, Med. Biolog. Eng. Comp., 37, 942, 1999.

Merla, A, Biomedical applications of functional infrared imaging, presented at the 21st Annual Meeting of Houston Society of Engineering in Medicine and Biology, Houston, TX, Feb 12-13, 2004.Merla, A., et al., Assessment of the effects of the varicocelectomy on the thermoregulatory control of the scrotum, Fertility and Sterility, 81,471, 2004.Merla, A., et al., Recording of the Sympathetic Thermal Response by means of Infrared Functional Imaging, in Proc. of the $25^{\text {th }}$ Annual International Conference of the IEEE Engineering in Medicine and Biology Society, Cancun, Mexico, Sept 17-21, 2003.

Merla, A., et al., Infrared Functional Imaging applied to the study of emotional reactions: preliminary results, in Proc. of the $4^{\text {th }}$ International Non Invasive Functional Source Imaging, Chieti, Italy, Sept, 9-13, 2003.

Merla, A. and Romani, G.L., Cutaneous blood flow rate mapping through functional infrared imaging, in Proc. of the World Congress of Medical Physics WC2003, Sidney, Aug, 24-29, 2003.

Merla, A., Cianflone, F., and Romani, G.L., Cutaneous blood flow rate estimation through functional infrared imaging analysis, in Proc. of the 5th International Federation of Automatic Control Symposium on Modelling and Control in Biomedical Systems, Melbourne, Aug, 19-23, 2003.

Merla, A., et al., Raynaud's Phenomenon: infrared functional imaging applied to diagnosis and drugs effects, Int. J. Immun. Pharm., 15, 41, 2002.

Merla, A., et al., Use of Infrared Functional Imaging to detect impaired thermoregulatory control in men with asymptomatic varicocele, Fertility and Sterility, 78, 199, 2002.

Merla, A., et al., Infrared Functional Imaging Applied to Raynaud's Phenomenon, IEEE Eng. Med. Biol. Mag., 21, 73, 2002.

Merla, A., et al., Quantifying the Relevance and Stage of Disease with the Tau image Technique. IEEE Eng. Med. Biol. Mag., 21, 86, 2002

Merla, A., et al., Infrared Functional Imaging: Analysis of cutaneous temperature during exercise, in Proc.of the $24^{\text {th }}$ IEEE Engineering in Medicine and Biology Society Conference, Houston, Oct 23-25, 2002.
ASHRAE: Handbook Fundamentals SI Edition, ASHRAE, Atlanta, GA, 1985

Merla, A., et al., Time Recovery Image: a diagnostic image technique based on the Dynamic Digital Telethermography, Thermol. Intern., $10,142,2000$.

Merla, A., et al.,. Tau image: a diagnostic imaging techinque based on the dynamic digital telethermography, in Proc. of the WC2000 Chicago World Congress on Medical Physics and Biomedical Engineering and 22th International Conference of IEEE Engineering in Medicine and Biology Society, Digest of Papers CD, track 1,TUFXH, Jul 2000, Chicago.

Allen, E.V. and Brown, G.E., Raynaud's disease: a critical review of minimal requisites for diagnosis, Am. J. Med. Sci., 183,187, 1932.

Subcommittee for Scleroderma Criteria of the American Rheumatism Association Diagnostic and Therapeutic Criteria Committee, Preliminary criteria for the classification of systemic sclerosis (scleroderma), Arthritis Rheum., 23,581,1980.

O'Reilly, D., et al., Measurement of cold challenge response in primary Raynaud's phenomenon and Raynaud's phenomenon associated with systemic sclerosis, Ann. Rheum. Dis., 51,1193, 1992. Clarks, S., et al., The distal-dorsal difference as a possible predictor of secondary Raynaud's phenomenon, J. Rheumatol., 26,1125, 1999. Schuhfried, O., et al., Thermographic parameters in the diagnosis of Secondary Raynaud's Phenomenon, Arch. Phys. Med. Rehabil., 81,495, 2000.

Ring, E.F.J., Cold Stress test for the hands, in The thermal image in Medicine and Biology, Uhlen Verlag, Ed., Wien, 1995.

Merla, A., et al., Combined approach to the initial stage Raynaud's phenomenon diagnosis by means of dynamic digital telethermography, capilloroscopy and pletismography: preliminary findings, Med. Biol. Eng. Comp., 37, 992, 1999.

Shitzer, A., et al., Lumped parameter tissue temperature-blood perfusion model of a cold stressed finger, J. Appl. Physiol., 80,1829, 1996.

Cooke, E.D., et al., Reflex sympathetic dystrophy and repetitive strain injury: temperature and microcirculatory changes following mild cold stress, J. Royal. Soc. Med., 86,690, 1993.

Di Benedetto, M., Regional hypothermia in response to minor injure, Am. J. Phys. Med. Rehabil., 75,270, 1996.

Garagiola, U., Use of telethermography in the management of sports injuries, Sports Med., 10, 267, 1990.

Maricq, H.R., et al., Diagnostic potential of in vivo capillary microscopy in scleroderma and related disorders, Arthr. Rheum., 23,183,1980.

Rodnan, G.P., Myerowitz, R.I., and Justh, G.O., Morphological changes in the digital arteries of patients with progressive systemic sclerosis and Raynaud Phenomenon, Medicine, 59,393,1980.

Tucker, A., Infrared thermographic assessment of the human scrotum, Fertil. Steril., 74,802, 2000.

Mieusset, R. and Bujan, L., Testicular heating and its possible contributions to male infertility: a review, Int. J. Andr., 18,169, 1995.

Trum, J. W., The value of palpation, varicoscreen contact thermography and colour Doppler ultrasound in the diagnosis of varicocele, Hum. $\quad 1996$. 